\title{
SIMULAÇÕES CFD EM REGIME TRANSIENTE PARA ALOCAÇÃO E CLASSIFICAÇÃO DE DETECTORES DE GASES INFLAMÁVEIS EM UNIDADE DE PROCESSA- MENTO DE PETRÓLEO OFFSHORE
}

\author{
J. M. BARCELOS, T. S. KLEIN e R. A. MEDRONHO \\ Universidade Federal do Rio de Janeiro, Escola de Química \\ E-mail para contato: joao.barcelos@eq.ufrj.br
}

\begin{abstract}
RESUMO - Na atividade offshore existem riscos associados a vazamentos de gás na planta de processo que causam preocupação com relação aos danos e às consequências que podem ser gerados. Neste trabalho, empregou-se a Fluidodinâmica Computacional na predição do comportamento da dispersão de gases leves a partir de um ponto de vazamento no sistema de compressão de gases. As condições operacionais, layout da unidade e condições ambientais presentes em sistemas reais foram levadas em consideração. Foram simulados 36 casos de vazamento em regime transiente avaliando-se o desenvolvimento de cada simulação com o tempo. Para classificar os sensores, foi proposta uma correlação para avaliar a efetividade de detecção dos mesmos. Com base nisso, propôs-se malhas de detecção capazes de identificar $100 \%$ dos casos em diferentes instantes de tempo.
\end{abstract}

\section{INTRODUÇÃO}

Dados estatísticos do HSE (Health \& Safety Executive) mostram que aproximadamente $40 \%$ dos vazamentos gasosos em ambiente offshore são detectados pelo sistema de detecção de gases da plataforma, sendo o restante identificado por outros tipos de sistemas de detecção. Nesse contexto, a determinação ótima da localização dos detectores de gases inflamáveis pode aumentar essa porcentagem e demonstra que é essencial entender como os gases se dispersam juntamente com o regime de vento e ventilação influenciados pela geometria da plataforma.

O posicionamento de detectores é normalmente baseado nas recomendações de normas, que o fazem de maneira vaga e imprecisa, de modo que os sensores sejam localizados próximos a possíveis pontos de vazamentos.

Simulações em CFD têm dado importantes contribuições em análises de segurança em diversas áreas. Essa ferramenta se destaca como uma alternativa economicamente viável e flexível. Muitos autores consideram os efeitos transientes na investigação do desenvolvimento temporal da dispersão gasosa estabelecendo posições baseadas no tempo de identificação do vazamento. Neste contexto, o presente trabalho propõe o estudo do comportamento transiente de nuvens de gás inflamável geradas por vazamento em uma unidade offshore através de simulações numéricas com CFD. A metodologia proposta define um sistema de detecção dos gases inflamáveis para o cenário de vazamento com o intuito de minimizar o número total de 


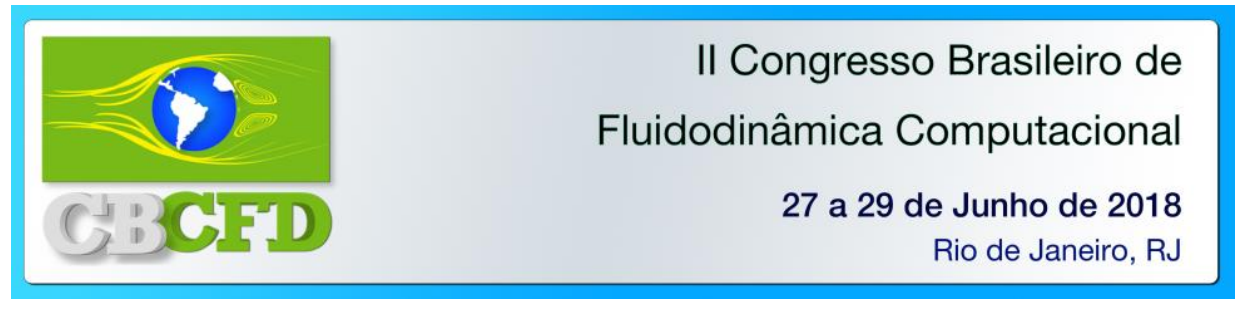

instrumentos mantendo-se uma confiabilidade razoável. Dessa forma, pretende-se identificar a importância de informações que só se pode obter no regime transiente, como o tempo de detecção e o tempo no qual a dispersão gasosa entra em equilíbrio com a ventilação ambiente. Além disso, propõe-se um parâmetro denominado de Efetividade de Detecção que estima a importância de cada dispositivo no sistema de detecção como um todo.

\section{METODOLOGIA}

De acordo com HSE (2002) os equipamentos que oferecem maior taxa de falhas são os que constituem o sistema de compressão dos gases. Assim, um compressor típico em condições de operação de pressão em $150 \mathrm{kgf} / \mathrm{cm}^{2}$ e temperatura de $128^{\circ} \mathrm{C}$ foi selecionado para o estudo. A geometria utilizada foi adaptada a partir das plataformas de petróleo em funcionamento e trata-se de um FPSO genérico com ancoragem do tipo Turret interno, conforme Figura 1. O módulo de compressão de gases é destacado na cor laranja.

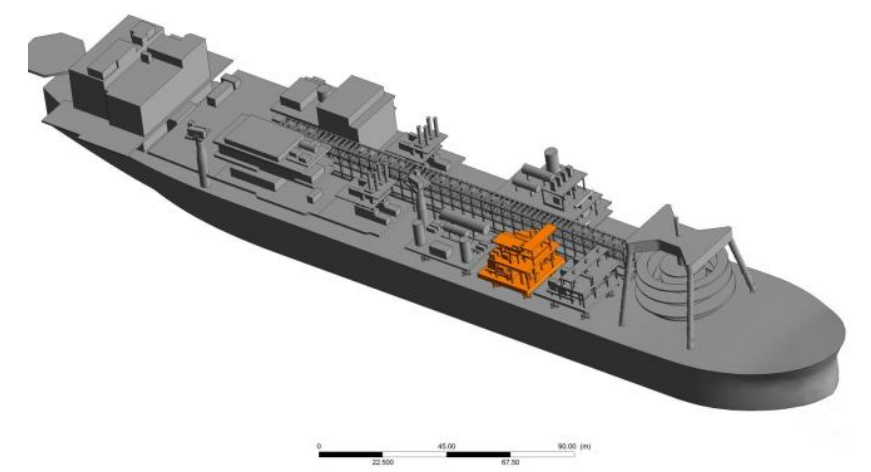

Figura 1 - Vista tridimensional do modelo geométrico.

O sistema de ancoragem do tipo Turret permite que a unidade fique alinhada de acordo com a resultante das cargas ambientais (Reyes, 2012). Neste caso, a carga ambiental considerada no estudo refere-se ao vento mais frequente (vento Nordeste - NE) que atinge a plataforma de frente, a $0^{\circ}$. Como esta rotação não ocorre de forma instantânea, considera-se uma variação nas direções de incidência do vento para $+30^{\circ}$ e $-30^{\circ}$.

Foi selecionado um ponto de vazamento para análise transiente, realizando-se 6 direções de jato $( \pm X, \pm Y$ e $\pm Z), 3$ direções de vento $\left(0^{\circ},+30^{\circ}\right.$ e $\left.-30^{\circ}\right)$ e 2 intensidades de vento (condição de calmaria de $0,5 \mathrm{~m} / \mathrm{s}$ e velocidade de vento mais frequente de $6,5 \mathrm{~m} / \mathrm{s}$ ) resultando em 36 simulações.

Para as simulações, considerou-se a composição do gás típica à do gás processado nesses sistemas. A vazão dos jatos foi determinada através de cálculos para escoamento subsônico após expansão isentrópica dos gases e inseridas como ponto fonte. Os valores utilizados foram de taxa de vazamento de $0,70 \mathrm{~kg} / \mathrm{s}$ (referente ao diâmetro do furo de 7,6 mm) e velocidade de saída de 122,5 m/s (modelo de fonte efetiva) calculados de acordo com TNO (2005) e Birch et al. (1987).

A malha computacional utilizada é tetraédrica e tem em torno de 15.000 .000 de elementos. Foi utilizado um passo de tempo de 1 s e o tempo total de simulação foi de 100 s. O 


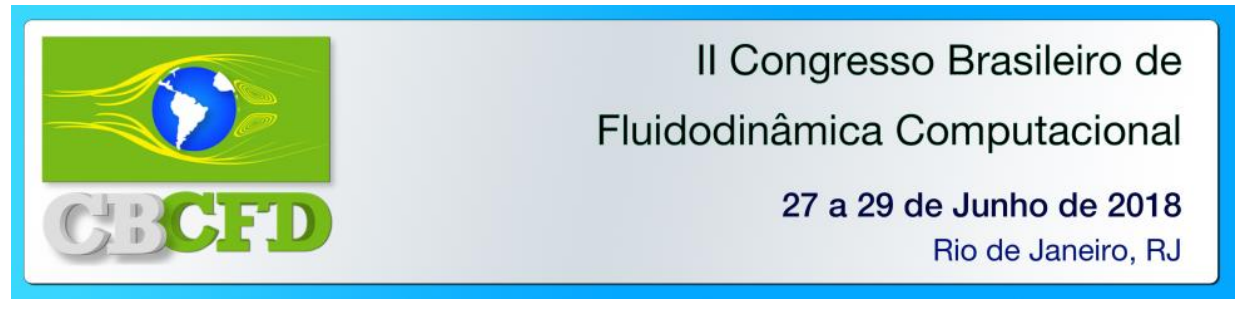

critério de convergência para as simulações foi de $10^{-4}$ para os resíduos médios. Testes de malhas e passo de tempo foram realizados para assegurar a confiabilidade dos resultados, bem como testes para resíduo e tempo necessário para assegurar que o estado estacionário fosse atingido.

Utilizou-se como concentração de detecção o valor de $20 \%$ do Limite Inferior de Inflamabilidade conforme recomendações de segurança para este tipo de unidade (Norma Técnica Petrobras N-2914).

O software POND4, desenvolvido pela Bureau Veritas, foi utilizado para o cálculo de ponderações de concentrações de gases, resultando na combinação de diferentes cenários de vazamento e ordenando os pontos (nós) onde é possível detectar o maior número de vazamentos. Sendo assim, o POND4 auxiliou na identificação dos pontos que identificam o maior número de casos.

Na determinação do posicionamento de detectores, alguns parâmetros são relevantes, como o tempo, para tomada de decisão, e o volume das nuvens, para o dimensionamento de danos e consequências. Além disso, o número de cenários que um detector consegue identificar, também é relevante. Dessa forma, foi proposta a Equação 1 para classificar os sensores, representando a Efetividade de Detecção para um determinado detector:

$$
E_{i}=\frac{1}{N_{T}} \sum_{j=1}^{N_{i}} f_{j} \frac{V_{E E, j}}{V_{E E, \max }} \frac{t_{E E, j}-t_{i j}}{t_{E E, j}}
$$

onde $N_{T}$ é o número total de cenários; $N_{i}$ é o número de cenários que o detector $i$ identifica; $f_{j}$ é a frequência de ocorrência do cenário $j ; V_{E E, j}$ é o volume da pluma do cenário $j$ quando o estado estacionário é atingido, em $t_{E E, j} ; V_{E E, \max }$ é o maior volume de pluma no estado estacionário dentre os $N_{T}$ cenários; e $\mathrm{t}_{\mathrm{ij}}$ é o tempo para o detector $i$ identificar o cenário $j$.

\section{RESULTADOS}

Observou-se que os cenários mais críticos são aqueles com jato direcionado para o piso da unidade, gerando grande acúmulo dos gases inflamáveis e aumentando a possibilidade de ocorrer explosões e incêndios.

A Figura apresenta a vista de topo da nuvem detectável em 5, 30 e 100 segundos de simulação para o caso com direção de jato $+Y$, direção de vento em $0^{\circ}$ e condição de calmaria com velocidade de $0,5 \mathrm{~m} / \mathrm{s}$.

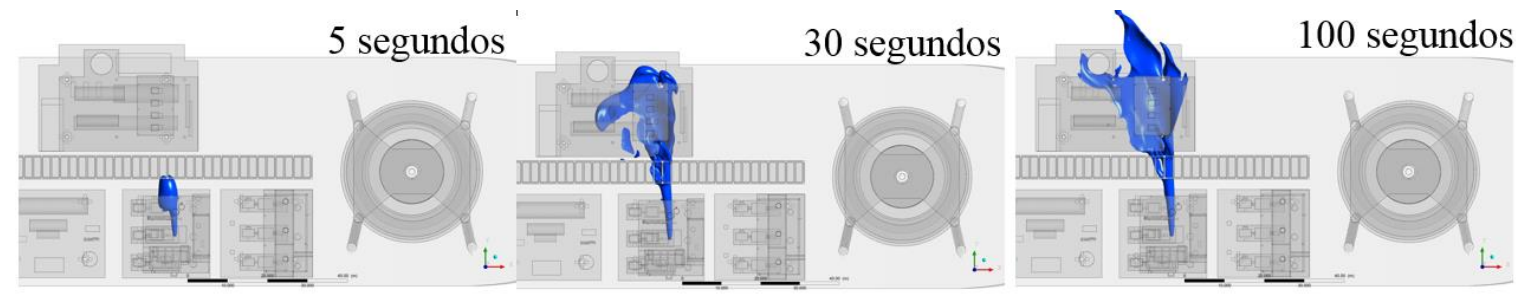

Figura 2 - Vista de topo da nuvem detectável do caso com direção de jato $+Y$, direção de vento em $0^{\circ}$ e condição de calmaria com velocidade de $0,5 \mathrm{~m} / \mathrm{s}$. 


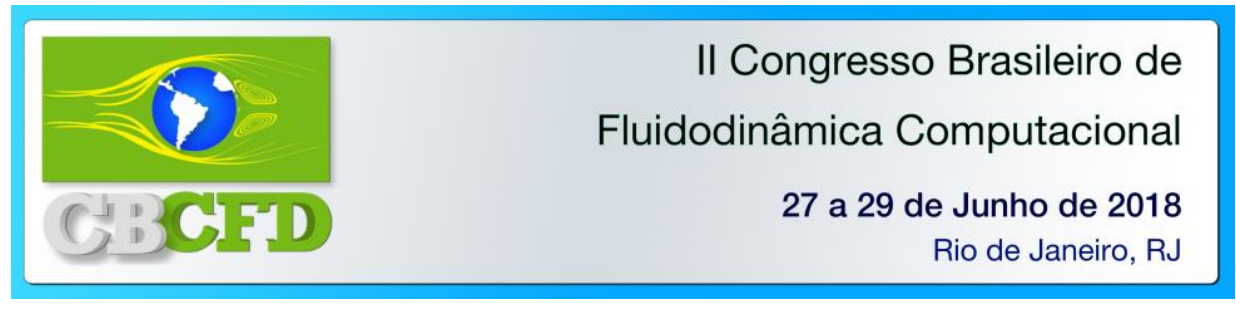

A metodologia de detecção indicou que 5 detectores são capazes de identificar $100 \%$ dos casos simulados após 100 segundos de simulação. Foram elaborados arranjos de detecção capazes de identificar todos os casos em 20 segundos e em 5 segundos de simulação. Os dois arranjos indicaram a necessidade de 5 detectores cada. Após análise das malhas de detecção propostas fica perceptível que ao diminuir o tempo de detecção dos casos simulados, o posicionamento dos sensores fica mais próximo do ponto de vazamento.

O cálculo da Efetividade de Detecção mostra que há uma relação entre os detectores que detectam o maior número de casos serem os mais efetivos, mas não garante que o que detecta mais casos é o mais efetivo. Por outro lado, os detectores mais rápidos, que detectam todos os cenários em até 5 segundos, não apresentaram necessariamente as melhores efetividades, o que, provavelmente, está associado ao volume da nuvem detectável gerada a partir de cada cenário.

\section{CONCLUSÃO}

A metodologia de detecção utilizada mostrou-se eficiente quanto à detecção de $100 \%$ dos casos analisados e à disposição geométrica dos detectores na planta de processo. Para detecção de todos os casos em 20 segundos e em 5 segundos também foram necessários cinco detectores, mostrando que o número mínimo de dispositivos para identificação em tempos diferentes não se altera necessariamente.

A equação da efetividade de detecção proposta mostrou-se coerente em relação aos parâmetros selecionados e demonstra que pode ser utilizada para classificação de todos os pontos observáveis do domínio de simulação, mas ajustes talvez sejam necessários para que se possa determinar a localização dos sensores apenas a partir da mesma.

\section{Agradecimentos}

A elaboração deste trabalho não teria sido possível sem a colaboração da Bureau Veritas, ao disponibilizar o software POND4.

\section{REFERÊNCIAS}

BIRCH, A. D., HUGHES, D. J. e SWAFFIELD, F. Velocity Decay of High Pressure Jets, Combust. Sci. and Tech., v. 52, p. 161-171, 1987.

FLECK, A. B. Dispersão de Gases Inflamáveis em Plataformas Offshore: Localização de Detectores, Dissertação de Mestrado, Escola de Química, UFRJ, Rio de Janeiro, 2008.

GOMES, E. G. Dispersão de Gases Inflamáveis em Unidades de Processamento de Petróleo: Otimização de Detectores, Dissertação de Mestrado, UFRJ/EQ, Rio de Janeiro, 2012.

Health \& Safety Executive. Offshore Hydrocarbon Releases Statistics and Analysis, 2002.

Reyes, M. C. Apostila de Tecnologia de Sistemas Oceânicos III, Rio de Janeiro, Construa, 2012.

TNO. Methods for the calculation of physical Effects (Yellow Book), The Netherlands Organization of Applied Scientific Research -, CPR14E, 3ª Ed, 2005. 